\title{
Myocardial injury after noncardiac surgery
}

\author{
P. J. Devereaux, MD, PhD, FRCPC (1) - Daniel Sessler, MD • Manoj Lalu, MD, PhD, FRCPC
}

Received: 6 January 2022/Revised: 6 January 2022 / Accepted: 6 January 2022 / Published online: 2 March 2022

(c) Canadian Anesthesiologists' Society 2022

In this issue of the Journal, Ko et al. report on the results of a large population-based historical cohort study of noncardiac surgical patients in Ontario. The investigators aimed to define patterns of postoperative troponin testing for detecting perioperative myocardial infarction. ${ }^{1}$ The principal finding was that there was substantial variation in troponin testing among hospitals, which the authors

P. J. Devereaux, MD, PhD, FRCPC $(\square)$

Department of Health Research Methods, Evidence, and Impact, McMaster University, Hamilton, ON, Canada

e-mail: pj.devereaux@phri.ca

Department of Medicine, McMaster University, Hamilton, ON, Canada

Population Health Research Institute, Hamilton, ON, Canada

Outcomes Research Consortium, Cleveland, OH, USA

D. Sessler, MD

Population Health Research Institute, Hamilton, ON, Canada

Outcomes Research Consortium, Cleveland, OH, USA

Department of OUTCOMES RESEARCH, Cleveland Clinic, Cleveland, OH, USA

M. Lalu, MD, PhD, FRCPC

Department of Anesthesiology and Pain Medicine, The Ottawa Hospital, Ottawa, ON, Canada

Blueprint Translational Research Group, Clinical Epidemiology Program, Regenerative Medicine Program, Ottawa Hospital Research Institute, Ottawa, ON, Canada

Department of Cellular and Molecular Medicine, University of Ottawa, Ottawa, ON, Canada suggest lends support to a more standardized approach to troponin testing. We use this opportunity to discuss the history of perioperative myocardial infarction and evolution to myocardial injury after noncardiac surgery (MINS); the prognosis, etiology, and management of MINS; and perioperative troponin monitoring.

\section{History of perioperative myocardial infarction and evolution to MINS}

Although angina pectoris was described in 1772, it was not until 1879 that pathologist Ludvig Hektoen proposed that myocardial infarction was due to coronary artery thrombosis. $^{2}$ Initial reports of myocardial infarction and associated mortality in the setting of noncardiac surgery started to occur in the late 1920s and early 1930s. In a 1938 article published in JAMA, Master et al. made the following astute observation: "the diagnosis of coronary artery occlusion following operation is frequently difficult, since the very severe pain ordinarily associated with this condition may be absent; it was present in only two fifths of our cases. This disparity may be accounted for, in part, by the liberal use of narcotics and sedatives after operation." 3 Although this statement was made eight decades ago, Master's warning that perioperative myocardial infarction frequently goes unrecognized remains as relevant today and was the underlying impetus for the evolution of perioperative myocardial infarction to MINS.

In 2014, the Vascular events In noncardiac Surgery patIents cOhort evaluatioN (VISION) investigators introduced the concept of MINS, proposed a definition, and undertook analyses to establish diagnostic criteria. ${ }^{4}$ Similar to Master, they recognized that perioperative myocardial infarction was a challenging diagnosis. 
Moreover, they showed that many patients sustain prognostically important ischemic myocardial injuries in the noncardiac surgery setting that will not fulfill the universal definition of myocardial infarction. Their proposed definition of MINS was "myocardial injury caused by ischemia (that may or may not result in necrosis), has prognostic relevance, and occurs during or within 30 days after noncardiac surgery." 4 This definition of MINS excluded perioperative myocardial injury caused by a documented nonischemic etiology (e.g., rapid atrial fibrillation, sepsis, pulmonary embolism, cardioversion). Analyses from the VISION cohort showed that myocardial injury believed to be due to ischemia (i.e., secondary to supply-demand mismatch or thrombosis) that did not fulfill the universal definition of myocardial infarction was independently associated with 30-day mortality (hazard ratio [HR], 3.30; 95\% confidence interval [CI], 2.26 to 4.81). ${ }^{4}$ These data supported a definition of MINS that was broader than myocardial infarction and encompassed both myocardial infarction and the prognostically relevant perioperative myocardial injuries due to ischemia that did not fulfill the definition of myocardial infarction.

Empiric research has moved past the proposed definition of MINS to establish functional diagnostic criteria for routine clinical use. Large epidemiological studies have explored various potential diagnostic criteria for MINS. Based on multivariable analyses that showed an association between proposed diagnostic criteria and 30-day mortality, these studies established the following diagnostic criteria for MINS: an elevated postoperative troponin level judged as resulting from myocardial ischemia (i.e., no evidence of nonischemic etiology), during or within 30 days after noncardiac surgery, and without the requirement of an ischemic feature (e.g., ischemic symptoms or ischemic electrocardiography changes).

Some have criticized the definition of MINS, noting if there is no evidence of a nonischemic etiology that this does not prove that myocardial ischemia was the cause. We have little doubt that the assumption that all myocardial injuries are due to myocardial ischemia, when there is no evidence of a nonischemic etiology, is false. Evidence, however, suggests that the vast majority of such cases are due to myocardial ischemia. One study of 40,004 patients (VISION) and another of 2,018 patients from Switzerland in which cardiologists and internists adjudicated the causes of perioperative troponin elevation based on all perioperative data identified a nonischemic etiology in only $10-15 \%$ of cases. ${ }^{5,6}$ Although they presumably missed some nonischemic cases, it seems unlikely they missed many nonischemic etiologies. Moreover, many clinical diagnoses require exclusion of alternative diagnoses, which is not unique to MINS.

Consider the following case. A patient has two hours of ischemic-sounding chest pain at home. After it resolves, they go to the emergency department. Their troponin is substantially elevated; their electrocardiogram (ECG) has no clear ischemic changes. The patient has no signs, symptoms, or laboratory evidence to suggest a nonischemic etiology for their troponin elevation. Although this patient meets the universal definition of myocardial infarction and is likely to be labeled as having had a myocardial infarction, there is no evidence that this event was unequivocally due to myocardial ischemia. It is possible that the patient had undetected rapid atrial fibrillation at home. This is also true even if the patient has angiography that shows a $70 \%$ lesion in a coronary artery. This case highlights how MINS is similar to myocardial infarction in that clinicians have to carefully determine whether there is evidence of a nonischemic etiology, as an alternative diagnosis to MINS or myocardial infarction. As always, clinical judgment is needed when making diagnoses.

\section{Prognosis}

The VISION study showed that MINS was associated with an increased risk of 30-day mortality (adjusted HR, 2.2; 95\% CI, 1.9 to 2.6) and the potential proportion of deaths attributable to MINS was $16 \% .^{5}$ The Swiss study of adults who had noncardiac surgery showed that MINS was associated with an increased risk of one-year mortality (adjusted HR, 1.48; 95\% CI, 1.07 to 2.06). ${ }^{6}$ MINS also portends a poor prognosis for future major cardiovascular events. The MANAGE trial included patients with MINS and during a mean follow-up of 16 months, patients randomized to placebo experienced cardiovascular death $(7 \%)$ and myocardial infarction $(5 \%)$ at rates much higher than patients who participated in nonsurgical randomized controlled trials of patients with known coronary artery disease or peripheral arterial disease. ${ }^{7}$

\section{Pathophysiology of MINS}

Myocardial injury after noncardiac surgery develops in a unique perioperative milieu of surgical trauma, inflammation, sympathetic stimulation, and metabolic dysregulation, all of which contribute to what could be regarded as iatrogenic ischemic injury. Indeed, observational studies have reported that well-recognized inflammatory and prothrombotic biomarkers correlate with the degree of myocardial injury. ${ }^{8,9}$ Advanced cardiac imaging studies have shown that the vast majority of patients with MINS have underlying coronary artery disease. $^{7}$ They also suggested that at least $75 \%$ of MINS events are due to supply-demand mismatch and the remainder are due to thrombosis. The degree of troponin 
elevation, ischemic symptoms, and ECG ischemic findings did not reliably differentiate supply-demand mismatch $v s$ thrombosis as the cause of MINS. This likely explains physicians' poor ability to identify whether MINS was due to supply-demand mismatch or thrombosis. ${ }^{10}$

\section{Perioperative troponin monitoring}

More than $90 \%$ of MINS is asymptomatic and will, thus, remain undetected without routine troponin screening. ${ }^{7}$ Consequently, recent guidelines, consensus documents, and scientific statements have recommended troponin screening in at-risk patients who have major noncardiac surgery. For example, the 2017 Canadian Cardiovascular Society guidelines recommended "daily troponin measurements for two to three days in patients with a baseline risk $>5 \%$ for cardiovascular death or nonfatal myocardial infarction at 30 days after surgery." ${ }^{11}$ In 2018, the Fourth Universal Definition of Myocardial Infarction consensus document stated that "postoperative troponin surveillance is recommended for high-risk individuals." 12 And in 2021, an American Heart Association scientific statement on MINS stated that "high-risk individuals should have serial cTn measurements during the first 48 to $72 \mathrm{hr}$ postoperatively." 13 Thus, there is a fairly broad consensus that troponin screening is indicated in at-risk patients, and recent work suggests that implementation is feasible and cost effective in the Canadian context. ${ }^{14}$

VISION cost-consequence analyses support perioperative troponin monitoring in patients $\geq 65 \mathrm{yr}$ of age or with a history of atherosclerotic disease. ${ }^{15}$ These simple criteria can facilitate implementation of monitoring strategies. The 2021 American Heart Association Scientific Statement on MINS suggests that troponin should be evaluated preoperatively, and in the first three postoperative days while patients remain hospitalized. The preoperative assessment is important because some patients have chronically elevated troponin measurements. Interpretation of postoperative troponin concentrations is, thus, facilitated by knowledge of the preoperative concentrations. Chronic troponin elevation is not a reason to postpone surgery.

\section{Management}

Although optimal management of MINS remains an area of investigation, accumulating evidence already points to several interventions that can improve outcomes. First, the poor short- and long-term prognosis of patients with MINS suggests that medical specialists should assess patients with MINS and follow them after hospital discharge.
Observational studies suggest that patients with MINS who are evaluated by a cardiologist have improved outcomes. $^{16,17}$ For patients with MINS, a medical specialist should explain to patients that they had myocardial injury and that their risk for future vascular events is elevated. The teachable moment should be used to discuss lifestyle enhancements that will reduce future risk including smoking cessation, healthy eating, and exercise.

Observational studies of patients with MINS support prescribing acetylsalicylic acid, a statin, and an angiotensin converting enzyme inhibitor. $^{7,18}$ The only trial of intervention for MINS $(N=1,754)$ showed that starting long-term dabigatran $110 \mathrm{mg}$ twice daily reduced the relative risk for subsequent major vascular events by $28 \%$ without increasing the risk of life-threatening, major, or critical organ bleeding. ${ }^{19}$ Patients should, therefore, be considered for dabigatran therapy.

\section{Conclusions}

The clinical entity of MINS evolved from observations regarding the challenges associated with the diagnosis of perioperative myocardial infarction (e.g., infrequent ischemic symptoms) and data related to the poor prognosis of perioperative myocardial injuries due to ischemia that does not fulfill the definition of myocardial infarction. Patients with MINS are at higher risk of cardiovascular death and myocardial infarction in the subsequent 16 months than nonsurgical patients with known coronary artery disease or peripheral arterial disease. Myocardial injury after noncardiac surgery is due to supply-demand mismatch or thrombosis. Because of the prognostic implications of MINS and available management options, current guidelines suggest that patients at risk of MINS (i.e., $\geq 65 \mathrm{yr}$ of age or with a history of atherosclerotic disease) should have troponin measured for the first three inpatient days after noncardiac surgery. Moreover, these guidelines also recommend that patients with MINS should be seen and followed by a medical specialist and that secondary cardiovascular prophylactic interventions should be initiated. Future work helping to optimize the diagnosis, prognosis, and management of MINS remains a priority for the $\geq 100$ million adults at risk of MINS who have noncardiac surgery annually.

\section{La lésion myocardique après une chirurgie non cardiaque}

Dans ce numéro du Journal, Ko et coll. rapportent les résultats d'une vaste étude de cohorte historique basée sur 
une population de patients chirurgicaux non cardiaques en Ontario. Les chercheurs s'étaient donné comme objectif de définir les habitudes de dosage des troponines postopératoires pour détecter l'infarctus du myocarde périopératoire. ${ }^{1}$ Leur principale conclusion était qu'il existait une variation substantielle dans le dosage des troponines entre les hôpitaux, ce qui, selon les auteurs, justifie l'adoption d'une approche plus standardisée. Nous profitons de cette occasion pour discuter de l'histoire de l'infarctus du myocarde périopératoire et de son évolution vers une lésion myocardique après une chirurgie non cardiaque (ou MINS, pour myocardial injury after noncardiac surgery); du pronostic, de l'étiologie et de la prise en charge de la MINS; et du monitorage périopératoire des troponines.

\section{Histoire de l'infarctus du myocarde périopératoire et évolution vers la MINS}

Bien que l'angine de poitrine ait été décrite en 1772, ce n'est qu'en 1879 que le pathologiste Ludvig Hektoen suggéra que l'infarctus du myocarde serait dû à une thrombose de l'artère coronaire. ${ }^{2}$ Les premiers comptes rendus d'infarctus du myocarde et de la mortalité qui y est associée dans le cadre de chirurgies non cardiaques ont commencé à apparaître à la fin des années 1920 et au début des années 1930. Dans un article de 1938 publié dans le JAMA, Master et coll. présentaient l'observation judicieuse suivante : « le diagnostic d'occlusion de l'artère coronaire après une opération est souvent difficile, car la douleur très intense habituellement associée à cette condition peut être absente; elle n'était présente que dans les deux cinquièmes de nos cas. Cette disparité peut s'expliquer, en partie, par l'usage libéral d'agents narcotiques et de sédatifs après l'opération $»{ }^{3}$ Bien que cette déclaration ait été faite il y a quatre-vingts ans, l'avertissement de Master selon lequel l'infarctus périopératoire du myocarde passe souvent inaperçu demeure tout aussi pertinent aujourd'hui, et a donné l'impulsion sous-jacente à l'évolution de l'infarctus du myocarde périopératoire vers la MINS.

En 2014, les chercheurs de l'étude VISION (Vascular events In noncardiac Surgery patIents cOhort evaluatioN) sur les événements vasculaires en chirurgie non cardiaque ont introduit le concept de MINS, proposé une définition et entrepris des analyses pour établir des critères diagnostiques. ${ }^{4}$ Tout comme Master, ces chercheurs ont reconnu que l'infarctus du myocarde périopératoire était un diagnostic difficile à poser. De plus, ils ont montré que, dans le cadre de chirurgies non cardiaques, de nombreux patients souffraient de lésions myocardiques ischémiques importantes d'un point de vue pronostique, tout en ne répondant pas à la définition universelle de l'infarctus du myocarde. Leur suggestion de définition de la MINS était « une lésion myocardique causée par une ischémie (qui peut ou non entraîner une nécrose), a une pertinence pronostique et survient pendant ou dans les 30 jours suivant une chirurgie non cardiaque. ${ }^{4}$ Cette définition de la MINS excluait les lésions myocardiques périopératoires causées par une étiologie non ischémique documentée (par ex. une fibrillation auriculaire rapide, un sepsis, une embolie pulmonaire, une cardioversion). Les analyses de la cohorte de l'étude VISION ont montré que les lésions myocardiques que l'on croyait être dues à une ischémie (c.à-d. secondaire à une inadéquation entre l'offre et la demande ou à une thrombose) qui ne correspondaient pas à la définition universelle de l'infarctus du myocarde étaient indépendamment associées à une mortalité à 30 jours (rapport de risque [RR], 3,30; intervalle de confiance à 95 $\%$ [IC], 2,26 à 4,81). ${ }^{4}$ Ces données étayaient une définition de la MINS plus large que l'infarctus du myocarde seul, une définition qui engloberait à la fois l'infarctus du myocarde et les lésions myocardiques périopératoires pertinentes d'un point de vue pronostique dues à une ischémie, mais qui ne correspondaient pas à la définition de l'infarctus du myocarde.

La recherche empirique a aujourd'hui dépassé la définition proposée de la MINS et a établi des critères diagnostiques fonctionnels destinés à une utilisation clinique de routine. De vastes études épidémiologiques ont exploré divers critères diagnostiques potentiels pour la MINS. Sur la base d'analyses multivariées qui ont montré une association entre les critères diagnostiques proposés et la mortalité à 30 jours, ces études ont établi les critères diagnostiques suivants pour la MINS : un taux élevé de troponines postopératoires jugé comme résultant d'une ischémie myocardique (c.-à-d. aucun signe d'étiologie non ischémique), pendant ou dans les 30 jours suivant une chirurgie non cardiaque, et sans exigence d'une caractéristique ischémique (p. ex., symptômes ischémiques ou changements électrocardiographiques ischémiques).

Certains ont critiqué la définition de la MINS, notant que, même s'il n'y a aucun signe d'une étiologie non ischémique, cela ne prouve pas que l'ischémie myocardique est la cause. Nous avons peu de doute que l'hypothèse selon laquelle toutes les lésions myocardiques sont dues à une ischémie myocardique, alors qu'il n'y a aucun signe d'étiologie non ischémique, est erronée. Les données probantes, cependant, suggèrent que la grande majorité de ces cas sont dus à une ischémie myocardique. Une étude portant sur 40004 patients (VISION) et une autre sur 2018 patients suisses, dans lesquelles des cardiologues et des internistes ont évalué les causes de l'élévation périopératoire des troponines sur la base de toutes les données périopératoires, ont identifié une 
étiologie non ischémique dans seulement 10 à $15 \%$ des cas. ${ }^{5,6}$ Bien qu'elles aient probablement manqué certains cas non ischémiques, il semble peu probable que ces études aient manqué un nombre important d'étiologies non ischémiques. De plus, de nombreux diagnostics cliniques nécessitent l'exclusion de diagnostics alternatifs, ce qui n'est pas unique au cas de la MINS.

Considérons le cas suivant. Un patient subit des douleurs thoraciques ressemblant à de l'ischémie durant deux heures à la maison. Une fois ses douleurs résolues, il se rend à l'urgence. Ses troponines sont considérablement élevées; son électrocardiogramme (ECG) ne montre pas de changements ischémiques clairs. Le patient ne présente aucun signe, symptôme ou test de laboratoire suggérant une étiologie non ischémique à l'élévation de ses troponines. Bien que ce patient réponde à la définition universelle de l'infarctus du myocarde et soit susceptible d'être étiqueté comme ayant subi un infarctus du myocarde, il n'y a aucune preuve que cet événement était sans équivoque dû à une ischémie myocardique. Il est possible que le patient ait subi une fibrillation auriculaire rapide non détectée à la maison. Cela est également vrai, même si l'angiographie du patient montre une lésion de $70 \%$ dans une artère coronaire. Ce cas met en évidence comment une MINS est similaire à un infarctus du myocarde, en ce sens que les cliniciens doivent déterminer avec soin s'il existe des signes d'une étiologie non ischémique comme diagnostic alternatif à la MINS ou à l'infarctus du myocarde. Comme toujours, le jugement clinique est nécessaire pour poser un diagnostic.

\section{Pronostic}

L'étude VISION a montré que la MINS était associée à un risque accru de mortalité à 30 jours (RR ajusté, 2,2; IC $95 \%, 1,9$ à 2,6 ) et que la proportion potentielle de décès attribuables à la MINS était de $16 \% .^{5}$ L'étude suisse portant sur des adultes ayant bénéficié d'une chirurgie non cardiaque a montré que la MINS était associée à un risque accru de mortalité à un an (RR ajusté, 1,48; IC $95 \%, 1,07$ à $2,06) .{ }^{6}$ La MINS laisse également présager un mauvais pronostic pour de futurs événements cardiovasculaires majeurs. L'étude MANAGE a inclus des patients atteints de MINS et, au cours d'un suivi moyen de 16 mois, les patients randomisés au placebo ont subi des taux beaucoup plus élevés de décès cardiovasculaires $(7 \%)$ et d'infarctus du myocarde $(5 \%)$ que les patients ayant participé à des études randomisées contrôlées non chirurgicales portant sur une coronaropathie connue ou une maladie artérielle périphérique. $^{7}$

\section{Physiopathologie des MINS}

Une lésion myocardique après une chirurgie non cardiaque survient dans un cadre périopératoire unique composé d'une combinaison de traumatisme chirurgical, d'inflammation, de stimulation sympathique et de dérèglement métabolique, lesquels contribuent tous à ce qui pourrait être considéré comme une lésion ischémique iatrogène. En effet, des études observationnelles ont rapporté que des biomarqueurs inflammatoires et prothrombotiques bien reconnus étaient corrélés au degré de lésion myocardique. ${ }^{8,9}$ Des études d'imagerie cardiaque avancée ont montré que la grande majorité des patients atteints de MINS souffraient de coronaropathie sousjacente. ${ }^{7}$ Ces études ont également suggéré qu'au moins $75 \%$ des événements de MINS étaient dus à une inadéquation entre l'offre et la demande, alors que le reste était dû à une thrombose. Le degré d'élévation des troponines, les symptômes ischémiques et les résultats de l'ECG d'ischémie ne permettent pas de différencier de manière fiable l'inadéquation entre l'offre et la demande ou la thrombose comme cause de MINS. Cela explique probablement la faible capacité des médecins à déterminer si la MINS est due à une inadéquation entre l'offre et la demande ou à une thrombose. ${ }^{10}$

\section{Monitorage périopératoire des troponines}

Plus de $90 \%$ des MINS sont asymptomatiques et resteront donc non détectées sans dosage systématique des troponines. ${ }^{7}$ Pour cette raison, des lignes directrices récentes, des documents de consensus et des énoncés scientifiques ont recommandé le dosage des troponines chez les patients à risque bénéficiant d'une chirurgie non cardiaque majeure. Par exemple, les lignes directrices de 2017 de la Société canadienne de cardiologie recommandaient « des mesures quotidiennes des troponines pendant deux à trois jours chez les patients présentant un risque de base $>5 \%$ de décès cardiovasculaire ou d'infarctus du myocarde non fatal 30 jours après la chirurgie. ${ }^{11}$ En 2018, le document de consensus sur la quatrième définition universelle de l'infarctus du myocarde (Fourth Universal Definition of Myocardial Infarction) indiquait que « le monitorage postopératoire des troponines est recommandé pour les personnes à haut risque $»{ }^{12}$ Et en 2021, un énoncé scientifique de l'American Heart Association concernant les MINS déclarait que «les personnes à haut risque devraient avoir des mesures des cTn (troponines cardiaques) répétées au cours des 48 à 72 premières heures postopératoires $\gg .{ }^{13}$ Ainsi, le consensus est assez large sur le fait que le dosage des troponines est indiqué 
chez les patients à risque, et des travaux récents suggèrent que la mise en œuvre d'une telle mesure est réalisable et rentable dans le contexte canadien. ${ }^{14}$

Les analyses coûts-conséquences de l'étude VISION appuient le monitorage périopératoire des troponines chez les patients âgés de $\geq 65$ ans ou ayant des antécédents d'athérosclérose. ${ }^{15}$ Ces critères simples peuvent faciliter la mise en œuvre de stratégies de monitorage. La déclaration scientifique 2021 de l'American Heart Association sur les MINS suggère que les troponines devraient être évaluées en préopératoire et au cours des trois premiers jours postopératoires, pendant que les patients restent hospitalisés. L'évaluation préopératoire est importante car certains patients affichent des mesures de troponines chroniquement élevées. L'interprétation des concentrations de troponines postopératoires est ainsi facilitée en connaissant les concentrations préopératoires. L'élévation chronique des troponines ne constitue pas une raison de reporter une chirurgie.

\section{Prise en charge}

Bien que la prise en charge optimale d'une MINS demeure matière à recherche, l'accumulation de données probantes indique déjà plusieurs interventions qui peuvent améliorer les devenirs. Tout d'abord, le mauvais pronostic à court et à long terme des patients atteints de MINS suggère que les médecins spécialistes devraient évaluer les patients atteints de MINS et en faire le suivi après leur congé de l'hôpital. Des études observationnelles suggèrent que les patients atteints de MINS évalués par un cardiologue auront de meilleurs devenirs. ${ }^{16,17}$ Pour les patients atteints de MINS, un médecin spécialiste doit expliquer aux patients qu'ils ont souffert d'une lésion myocardique et que leur risque d'événements vasculaires futurs est élevé. Ce moment propice à l'enseignement devrait être utilisé pour discuter des améliorations des habitudes de vie qui réduiront les risques futurs, y compris l'abandon du tabac, une alimentation équilibrée et l'exercice.

Les études observationnelles de patients atteints de MINS endossent la prescription d'acide acétylsalicylique, d'une statine et d'un inhibiteur de l'enzyme de conversion de l'angiotensine. ${ }^{7,18} \mathrm{La}$ seule étude interventionnelle portant sur les MINS $(n=1754)$ a montré que le fait d'amorcer un traitement à long terme de $110 \mathrm{mg}$ de dabigatran deux fois par jour réduisait le risque relatif d'événements vasculaires majeurs ultérieurs de $28 \%$, sans augmenter le risque de saignements d'organes majeurs, potentiellement fatals ou critiques. ${ }^{19}$ Il convient dès lors d'envisager un traitement à base de dabigatran pour ces patients.

\section{Conclusion}

En tant qu'entité clinique, la lésion myocardique après une chirurgie non cardiaque a évolué, en se fondant d'abord sur les observations concernant les défis associés au diagnostic de l'infarctus du myocarde périopératoire (par exemple, des symptômes ischémiques peu fréquents) puis sur les données liées au mauvais pronostic des lésions myocardiques périopératoires dues à une ischémie, mais qui ne répondaient pas à la définition de l'infarctus du myocarde. Les patients atteints de MINS courent un risque plus élevé de décès cardiovasculaire et d'infarctus du myocarde au cours des 16 mois suivant leur opération que les patients non chirurgicaux atteints d'une maladie coronarienne connue ou d'une maladie artérielle périphérique. La lésion myocardique après une chirurgie non cardiaque est due à une inadéquation entre l'offre et la demande ou à une thrombose. En raison des implications pronostiques de la MINS et des options de prise en charge disponibles, les lignes directrices actuelles suggèrent que les troponines soient dosées chez les patients à risque de MINS (c.-à-d. $\geq$ de 65 ans ou ayant des antécédents de maladie athéroscléreuse) pendant les trois premiers jours d'hospitalisation après une chirurgie non cardiaque. De plus, ces lignes directrices recommandent également que les patients atteints de MINS soient vus et suivis par un médecin spécialiste et que des interventions prophylactiques cardiovasculaires secondaires soient amorcées. Les travaux futurs visant à optimiser le diagnostic, le pronostic et la prise en charge des MINS restent une priorité pour les $\geq 100$ millions d'adultes à risque de MINS qui subissent chaque année une chirurgie non cardiaque.

Disclosures None.

Funding statement None.

Editorial responsibility This submission was handled by Dr. Stephan K. W. Schwarz, Editor-in-Chief, Canadian Journal of Anesthesia.

Déclaration Aucune.

Déclaration de financement Aucune.

Responsabilité éditoriale Ce manuscrit a été traité par Dr Stephan $\mathrm{K}$. W. Schwarz, rédacteur en chef, Journal canadien d'anesthésie.

\section{References}

1. Azizi PM, Wijeysundera DN, Wijeysundera HC, et al. Troponin testing after non-cardiac surgery: variation and factors associated 
with testing. Can J Anesth 2022; DOI: https://doi.org/10.1007/ s12630-022-02219-y.

2. Nabel EG, Braunwald E. A tale of coronary artery disease and myocardial infarction. N Engl J Med 2012; 366: 54-63.

3. Master AM, Dack S, Jaffe HL. Postoperative coronary artery occlusion. JAMA 1938; 110: 1415-8.

4. Botto F, Alonso-Coello P, Chan MT, et al. Myocardial injury after noncardiac surgery: a large, international, prospective cohort study establishing diagnostic criteria, characteristics, predictors, and 30-day outcomes. Anesthesiology 2014; 120: 564-78.

5. Vascular Events in Noncardiac Surgery Patients Cohort Evaluation Study Investigators; Spence J, LeManach Y, Chan $M T$, et al. Association between complications and death within 30 days after noncardiac surgery. CMAJ 2019; 191: E830-7.

6. Puelacher C, Lurati Buse G, Seeberger D, et al. Perioperative myocardial injury after noncardiac surgery: incidence, mortality, and characterization. Circulation 2018; 137: 1221-32.

7. Devereaux PJ, Szczeklik W. Myocardial injury after non-cardiac surgery: diagnosis and management. Eur Heart J 2020; 41: 3083-91.

8. Gorka J, Polok K, Iwaniec $T$, et al. Altered preoperative coagulation and fibrinolysis are associated with myocardial injury after non-cardiac surgery. Br J Anaesth 2017; 118: 713-9.

9. Ackland GL, Abbott TEF, Cain D, et al. Preoperative systemic inflammation and perioperative myocardial injury: prospective observational multicentre cohort study of patients undergoing non-cardiac surgery. Br J Anaesth 2019; 122: 180-7.

10. Borges FK, Sheth T, Patel A, et al. Accuracy of physicians in differentiating type 1 and type 2 myocardial infarction based on clinical information. CJC Open 2020; 2: 577-84.

11. Duceppe E, Parlow J, MacDonald P, et al. Canadian Cardiovascular Society Guidelines on perioperative cardiac risk assessment and management for patients who undergo noncardiac surgery. Can J Cardiol 2017; 33: 17-32.

12. Thygesen $K$, Alpert JS, Jaffe AS, et al. Fourth Universal Definition of Myocardial Infarction (2018). J Am Coll Cardiol 2018; 72: 2231-64.

13. Ruetzler K, Smilowitz NR, Berger JS, et al. Diagnosis and management of patients with myocardial injury after noncardiac surgery: a scientific statement from the American Heart Association. Circulation 2021; 144: e287-305.

14. McIsaac DI, Montroy J, Gagne S, et al. Implementation of the Canadian Cardiovascular Society guidelines for perioperative risk assessment and management: an interrupted time series study. Can J Anesth 2021; 68: 1135-45.

15. Buse GL, Manns B, Lamy A, et al. Troponin T monitoring to detect myocardial injury after noncardiac surgery: a costconsequence analysis. Can J Surg 2018; 61: 185-94.

16. Park J, Oh AR, Kwon JH, et al. Association between cardiologist evaluation and mortality in myocardial injury after non-cardiac surgery. Heart 2021; DOI: https://doi.org/10.1136/heartjnl-2021319511.

17. Hua A, Pattenden H, Leung M, et al. Early cardiology assessment and intervention reduces mortality following myocardial injury after non-cardiac surgery (MINS). J Thorac Dis 2016; 8: 920-4.

18. Gouda P, Wang X, McGillion M, Graham MM. Underutilization of perioperative screening for cardiovascular events after noncardiac surgery in Alberta. Can J Cardiol 2021; 37: 57-65.

19. Devereaux PJ, Duceppe E, Guyatt G, et al. Dabigatran in patients with myocardial injury after non-cardiac surgery (MANAGE): an international, randomised, placebo-controlled trial. Lancet 2018; 391: 2325-34.

Publisher's Note Springer Nature remains neutral with regard to jurisdictional claims in published maps and institutional affiliations. 